\title{
Conductive polymer binder for nano-silicon/graphite composite electrode in lithium-ion batteries towards a practical application
}

Hui Zhao,${ }^{\mathrm{a}}+$ Allen Du, ${ }^{\mathrm{b}}+$ Min Ling, ${ }^{\mathrm{a}}$ Vincent Battaglia, ${ }^{\mathrm{a}}$ and Gao Liu ${ }^{\mathrm{a}}$

${ }^{a}$ Applied Energy Materials Group, Energy Storage and Distributed Resources Division, Lawrence Berkeley National Laboratory, Berkeley, California, 94720, United States

${ }^{\mathrm{b}}$ University of Michigan, Ann Arbor, 48109, United States

*Tel.: +1-510-486-7207; fax: +1-510-486-7303; Email: gliu@lbl.gov (G. Liu)

† These authors contributed equally to this work

\begin{abstract}
The state-of-the-art graphite anode containing a small portion of silicon represents a promising way of applying high-capacity alloy anode in the next generation high energy density lithium-ion batteries. The conductive polymeric binders developed for $\mathrm{Si}$ anodes proved to be an effective binder for this graphite/nanoSi composite electrode. Without any acetylene black conductive additives in the electrode, a high areal capacity of above $2.5 \mathrm{mAh} / \mathrm{cm}^{2}$ is achieved during long-term cycling over 100 cycles. This conductive polymer-enabled graphite/nanoSi composite electrode exhibits high specific capacity and high $1^{\text {st }}$ cycle efficiency, which is a significant progress toward commercial application of Si anodes.
\end{abstract}

Keywords: conductive polymer binder, silicon nanoparticle, graphite, lithium-ion battery, practical application 


\section{Introduction}

As human civilization advances, global energy consumption steadily increases. This necessitates a green method to effectively store energy, such as using fuel cells, flows batteries, and lithium-ion batteries (LIBs).[1] State-of-the-art LIBs use graphite as anode, with a theoretical gravimetric specific capacity of about $370 \mathrm{mAh} / \mathrm{g}$. Alternative alloy anodes, such as silicon or tin, are shown as promising successors to graphite. Si has a gravimetric specific capacity over 10 times higher than graphite $(4200 \mathrm{mAh} / \mathrm{g})$, but a $300 \%$ volume expansion during lithiation prevents it from becoming commercially viable. The high volume changes during lithiation and delithiation cycles cause excessive electrolyte decomposition, leading to capacity decay. Another effect of the volume change is the difficulty to assemble electrodes with higher material loading for a stable cell performance. An area capacity of higher than $2.5 \mathrm{mAh} / \mathrm{cm}^{2}$ is necessary and highly desirable towards a practical application of these high capacity alloy anodes in a commercial cell. New binders have been developed in recent years to achieve a high material loading in the silicon based anode, a cross-linked water soluble polymeric binder[2] and a conductive polymer binder[3], along with other types of binders, have shown that the right binder chemistry is critical toward a high-performance silicon anode.

Typically a high content of polymer binder and conductive additives are needed to enable a stable cycling of Si anode.[4] However, this unacceptably decreases the energy density due to the excessive non-active materials used to maintain a good cell performance. An electronic conductive polymeric binder developed for $\mathrm{Si}$ anodes achieved very stable cycling of $\mathrm{Si}$ anode with good active material loading.[5] Since no conductive additives are used, a high content of active material content is achieved. A conductive polymer binder compatible with both graphite 
and nanoSi anodes are used to assemble a graphite/nanoSi composite electrode in this work. This conductive polymer-enabled composite electrode presents a promising opportunity for the practical application of high capacity alloy anodes for the next-generation lithium-ion batteries.

\section{Experimental}

All reagents were purchased from Sigma-Aldrich or TCI America and used without further purification. Synthesis of poly(1-pyrenemethyl methacrylate) binder was shown in a previous publication.[6] CGP-G8, purchased from ConocoPhillips, was used as the graphite anode. The nanoSi with a particle size of 50-70 nm is from Nanostructured and Amorphous Inc. Celgard 2400 separator is obtained from Celgard. Lithium-ion electrolyte were purchased from BASF, including $1 \mathrm{M} \mathrm{LiPF}_{6}$ in ethylene carbonate, diethyl carbonate $(\mathrm{EC} / \mathrm{DEC}=3 / 7 \mathrm{w} / \mathrm{w})$ containing $30 \mathrm{wt} \%$ fluoroethylene carbonate (FEC). 2325 coin cells were prepared using lithium metal as a counter electrode. Slurry preparation, electrode coating, and cell fabrication were completed using standard techniques in the literature.[7] SEM images of the composite electrode surface were collected with a JEOL JSM-7500F field emission scanning electron microscopy with an accelerating voltage of $15 \mathrm{kV}$ using the high vacuum mode at room temperature. High-resolution TEM images were obtained on a Philips CM200 field emission microscope operated at $200 \mathrm{kV}$ at the National Center for Electron Microscopy (NCEM) at Lawrence Berkeley National Laboratory (LBNL).

\section{Results and Discussion}

A pyrene-based homo-polymer poly(1-pyrenemethyl methacrylate) (PPy, Figure 1a) is synthesized and used as the conductive polymer binder for the graphite/nanoSi composite 
electrode. The graphite materials are composed of micron-size particles shown in Figure $1 \mathrm{~b}$. The micron-size particle with small surface area is the origin of its high Coulombic efficiency during cell cycling, which induces minimum electrolyte decomposition and formation solid electrolyte interphase (SEI).[8]

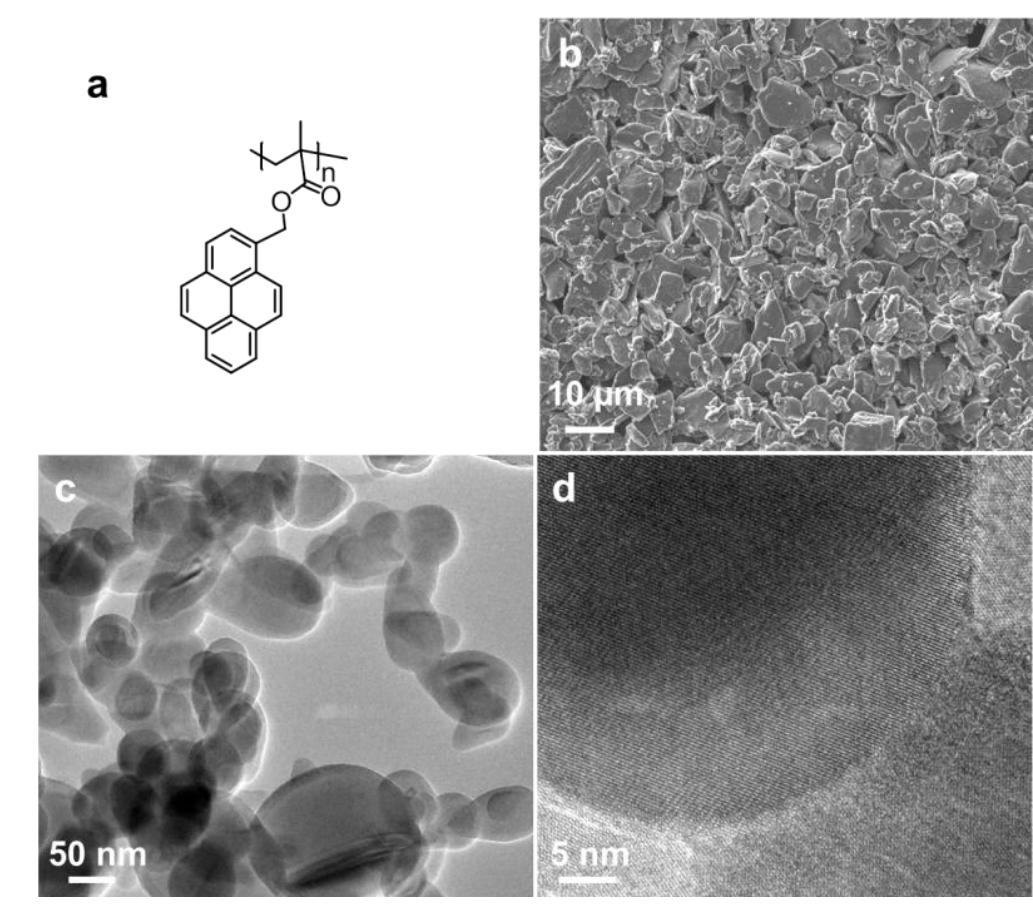

Figure 1. (a) Chemical structure of the PPy binder. (b) SEM image of the graphite particles. (c) and (d) High Resolution TEM (HRTEM) images of the nanoSi particles.

A typical nanoSi material is used to reduce the volume changes on the particle level and avoid particle cracking upon lithiation. The average particles size of the nanoSi is around 50-70 nm, with a high surface area of $\sim 50 \mathrm{~m}^{2} / \mathrm{g}$.[9] The nanoSi with high surface area needs significant electrolyte decomposition to form SEI and passivate the surface, the resulting low $1^{\text {st }}$ cycle coulombic efficiency hinders a practical application of the high capacity nanoSi anodes. Even with a particle size in the nano-domain, Figure $1 \mathrm{~d}$ still indicates crystalline silicon phase in this nanoSi particles. Overcoming the low initial efficiency is an intensive topic in recent literature.[10] The whole problem of stable cycling of Si anode is the $300 \%$ volume changes due to its high specific capacity (4200 mAh/g). However, compared to the state-of-the-art graphite anode (372 mAh/g), 
an anode with an intermediate specific capacity at 700-1000 mAh/g could significantly enhance the energy density in the full cell configuration. Thus, in this work we mix graphite and nanoSi with PPy binder during slurry preparation process, only $20 \mathrm{wt} \% \mathrm{Si}$ is used in the electrode assembly, which will only leads to $30 \%$ to $50 \%$ volume changes on the electrode level; on the other hand, the $70 \mathrm{wt} \%$ graphite material serves as a buffer to accommodate the volume changes on the electrode level, while maintaining a good electronic conduction channel throughout the whole electrode laminate.

a

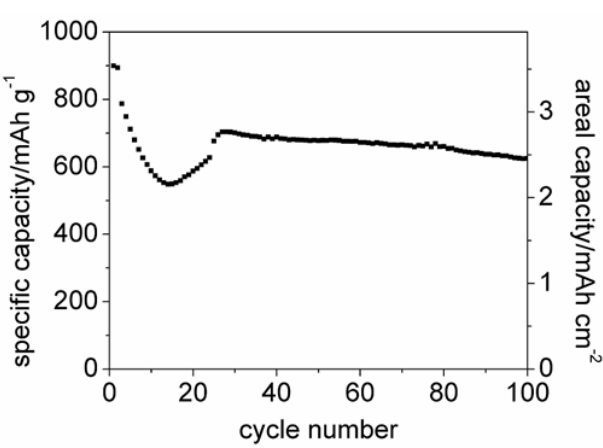

C

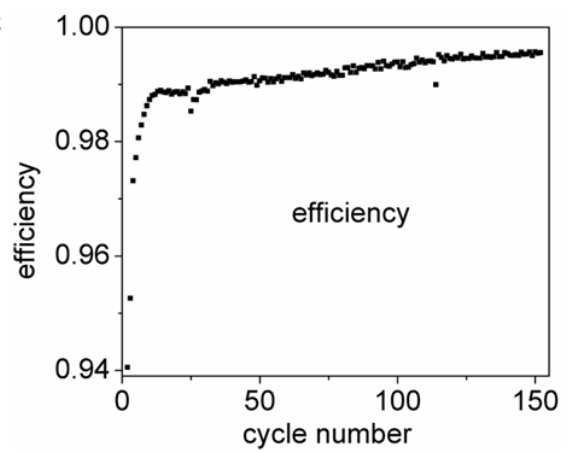

b

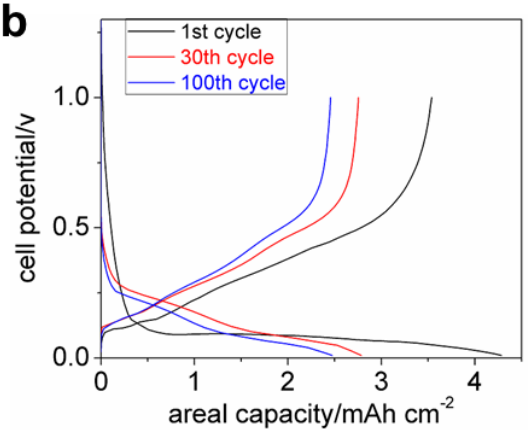

d

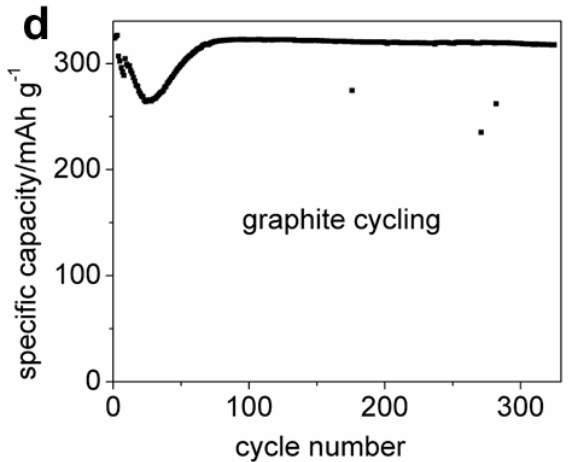

Figure 2. (a) Specific capacity (left $\mathrm{Y}$ axis) and areal capacity (right $\mathrm{Y}$ axis) vs. cycle number using 10 wt $\%$ PPy binder with $20 \mathrm{wt} \%$ nanoSi and $70 \mathrm{wt} \%$ graphite. The initial two cycles were at $\mathrm{C} / 25$, then $\mathrm{C} / 10$ for the long-term cycling. (b) $1^{\text {st }}, 30^{\text {th }}$, and $100^{\text {th }}$ cycle voltage curves of the PPy/graphite/nanoSi mixture electrode. (c) Coulombic efficiency vs. cycle number from the second cycle $\left(1^{\text {st }}\right.$ cycle efficiency is 69.79\%). (d) Cell performance of graphite electrode based on PPy binder at a C/10 rate.

Previous work proves that the conductive PPy binder enables a very stable cycling of nanoSi material.[3] PPy also enables a stable cycling performance of the graphite anode.[6] Figure 2d shows the cell performance based on $10 \mathrm{wt} \%$ PPy and $90 \mathrm{wt} \%$ graphite at a C/10 rate. Almost full specific is achieved for this graphite anode material (around $310 \mathrm{mAh} / \mathrm{g}$ for CGP-G8, 
ConocoPhillips), which is stable for several hundreds of cycles. Note that the initial decrease of specific capacity value in the initial 30 cycles is proposed due to electrolyte wetting, which self-recovers to a value of above $300 \mathrm{mAh} / \mathrm{g}$ after 50 cycles.

Targeting at a practical application in commercial cell, a relatively high loading electrode is assembled and tested. This specific cell shown in Figure $2 \mathrm{a}$ contains $0.87 \mathrm{mg}$ nanoSi $/ \mathrm{cm}^{2}$ and 3.06 mg graphite $/ \mathrm{cm}^{2}$. The left $\mathrm{Y}$ axis in Figure 2a is plotted as mAh per gram of graphite \& nanoSi, which exhibits twice as high as conventional graphite anode even after 100 cycles. Also, a signature areal capacity of $2.5 \mathrm{mAh} / \mathrm{cm}^{2}$ is achieved and maintained stable for more than 100 cycles. The corresponding voltage curves at $1^{\text {st }}$ cycle, $30^{\text {th }}$ cycle, and $100^{\text {th }}$ cycle are shown in Figure 2b. Consistent with the crystalline Si phase in the TEM morphology (Figure 1d), a large overpotential is shown in the $1^{\text {st }}$ cycle lithiation curve, corresponding to the amorphorization of the crystalline Si phase. The Si phase is kept amorphous in the following cycles, which is also reflected in the $30^{\text {th }}$ and $100^{\text {th }}$ cycle voltage curves.

\begin{tabular}{|c|c|c|c|c|}
\hline & & Graphite/PPy & nanoSi/PPy & nanoSi/graphite/PPy \\
\hline \multirow{2}{*}{$1^{\text {st }}$ cycle } & $Q_{\mathrm{c}}{ }^{\mathrm{a}}(\mathrm{mAh} / \mathrm{g})$ & 323.7 & 3482.4 & 899.9 \\
\hline & $\eta^{\mathrm{b}}(\%)$ & 87.12 & 69.79 & 82.59 \\
\hline \multirow{2}{*}{$30^{\text {th }}$ cycle } & $Q_{\mathrm{c}}{ }^{\mathrm{a}}(\mathrm{mAh} / \mathrm{g})$ & 266.8 & 2868.8 & 700.8 \\
\hline & $\eta^{\mathrm{b}}(\%)$ & 99.78 & 98.21 & 98.91 \\
\hline \multirow{2}{*}{$100^{\text {th }}$ cycle } & $Q_{\mathrm{c}}{ }^{\mathrm{a}}(\mathrm{mAh} / \mathrm{g})$ & 322.9 & 2907.6 & 625.1 \\
\hline & $\eta^{\mathrm{b}}(\%)$ & 99.83 & 98.93 & 99.39 \\
\hline
\end{tabular}

Table 1. Electrochemical parameters of PPy conductive binder with different anode materials.

The main electrochemical parameters of the cell performance using $10 \mathrm{wt} \%$ PPy binders with different anode materials are shown in Table 1. It shows that PPy conductive polymer is compatible with the conventional graphite anode, with a high $1^{\text {st }}$ cycle efficiency of $87.12 \%$. The 
efficiency value is enhanced to $99.83 \%$ after 100 cycles at a $\mathrm{C} / 10$ rate. A previous work established that PPy is a very good polymer binder for a long-term stable cycling of nanoSi anode,[6] with a typical $1^{\text {st }}$ cycle efficiency of less than $70 \%$. This low $1^{\text {st }}$ cycle efficiency is typical for a nano-material electrode, which is due to the excessive side reaction and SEI formation from the high surface area. The low efficiency value at $1^{\text {st }}$ cycle will consume excessive lithium in a full cell configuration, which is a major hurdle for the application of nanoSi anode. The compatibility of the PPy conductive polymer binder with both graphite and nanoSi is the foundation for the successful development of graphite/nanoSi mixture electrode. As shown in Table 1, mixing $20 \mathrm{wt} \% \mathrm{Si}$ and $70 \%$ into the electrode formulation maintains a specific capacity value twice as high as the graphite anode even at $100^{\text {th }}$ cycle, while enabling a $1^{\text {st }}$ cycle efficiency as high as $82.59 \%$. Simply mixing graphite with a small portion of nanoSi enhances the specific capacity while not sacrificing $1^{\text {st }}$ cycle efficiency, together with a high areal capacity of above 2.5 $\mathrm{mAh} / \mathrm{cm}^{2}$, this approach represents a good way of using high capacity alloy anode in a the commercial application.[11]

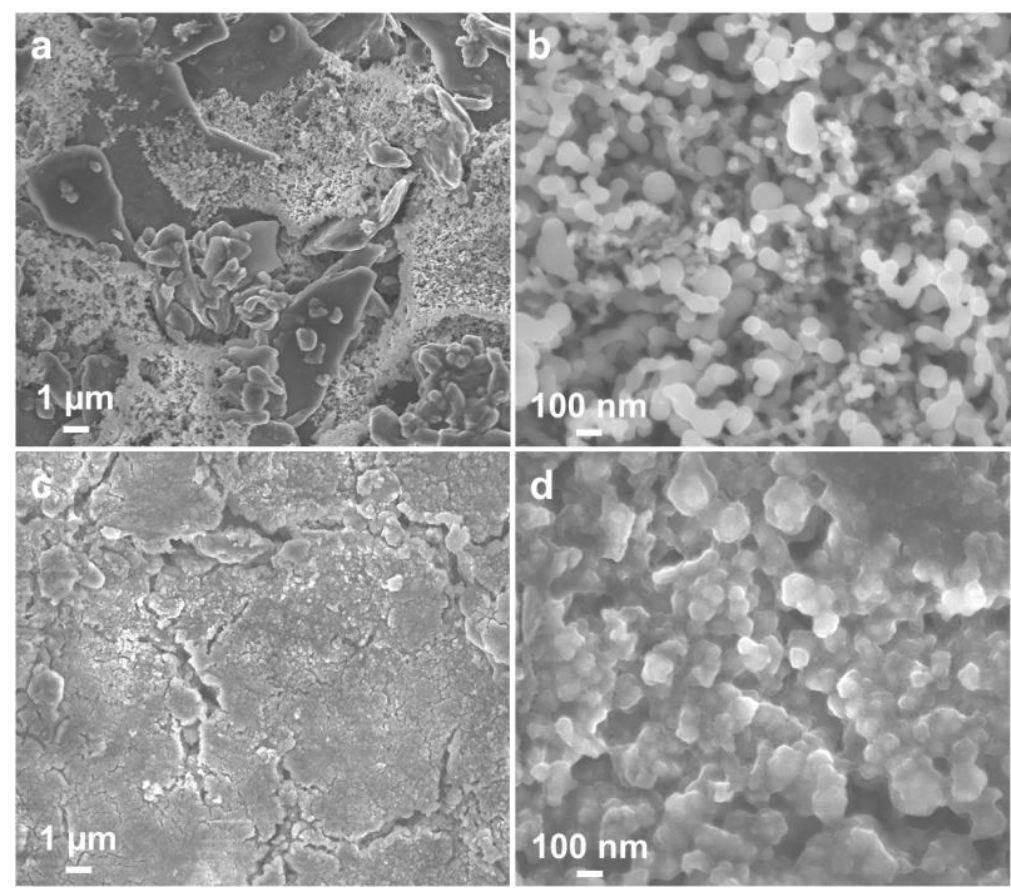


Figure 3. SEM image (a) (b) pristine electrodes and (c) (d) cycled electrodes (10 cycles after C/10).

The morphology of the pristine and cycled PPy/graphite/nanoSi electrode is shown in Figure

3. Although there is only $20 \mathrm{wt} \%$ nanoSi in the electrode, the tap density for this nanoSi is quite small and the actual volume of added nanoSi is significant compared to the micro-size graphite anode material with high tap density. Thus, in the top-view SEM image of the pristine electrode in Figure 3a, a large portion of the material in the image is nanoSi. An expanded view on the nanoSi part in Figure $3 b$ indicates good distribution of the nanoSi particles in the electrode with decent porosity. The electrolyte used in this work involves FEC additives, which results in a typical cycled electrode morphology shown in Figure 3c and 3d.

\section{Conclusions}

The state-of-the-art graphite anode containing a small portion of silicon represents a promising way of applying high-capacity alloy anode in the next generation high energy density lithium-ion batteries. Without any acetylene black conductive additives in the conductive polymer/graphite /nanoSi electrode, a high areal capacity of above $2.5 \mathrm{mAh} / \mathrm{cm}^{2}$ is achieved during long-term cycling over 100 cycles. This conductive polymer-enabled graphite/nanoSi composite electrode exhibits high specific capacity and high $1^{\text {st }}$ cycle efficiency, which is a significant progress toward commercial application of $\mathrm{Si}$ anodes.

\section{ASSOCIATED CONTENT}

\section{AUTHOR INFORMATION}

\section{Corresponding Author}

*E-mail: gliu@lbl.gov

\section{Notes}

The authors declare no competing financial interests. 


\section{ACKNOWLEDGEMENTS}

This work was funded by the Assistant Secretary for Energy Efficiency, Vehicle Technologies

Office of the U.S. Department of Energy (U.S. DOE) under the Advanced Battery Materials Research (BMR) and Applied Battery Research (ABR) Programs. TEM is performed at the National Center for Electron Microscopy. All these projects and facilities are supported by the Director, Office of Science, Office of Basic Energy Sciences, of the U.S. Department of Energy, under Contract \# DE-AC02-05 CH11231.

\section{References}

[1] J.B. Goodenough, Y. Kim, Challenges for Rechargeable Li Batteries, Chem. Mater. 22 (2010) 587-603.

[2] J. Song, M. Zhou, R. Yi, T. Xu, M.L. Gordin, D. Tang, Z. Yu, M. Regula, D. Wang, Adv. Functional Mater. 24 (2014) 5904-5910.

[3] G. Liu, S. Xun, N. Vukmirovic, X. Song, P. Olalde-Velasco, H. Zheng, V.S. Battaglia, L. Wang, W. Yang, Adv. Mater. 23 (2011) 4679-4683.

[4] S.D. Beattie, D. Larcher, M. Morcrette, B. Simon, J.-M. Tarascon, Si Electrodes for Li-Ion Batteries-A New Way to Look at an Old Problem, J. Electrochem. Soc. 155 (2008) A158-A163.

[5] H. Zhao, Z. Wang, P. Lu, M. Jiang, F. Shi, X. Song, Z. Zheng, X. Zhou, Y. Fu, G. Abdelbast, X. Xiao, Z. Liu, V.S. Battaglia, K. Zaghib, G. Liu, Nano Lett. 14 (2014) 6704-6710.

[6] S.-J. Park, H. Zhao, G. Ai, C. Wang, X. Song, N. Yuca, V.S. Battaglia, W. Yang, G. Liu, J. Am. Chem. Soc. 137 (2015) 2565-2571.

[7] G. Liu, H. Zheng, A.S. Simens, A.M. Minor, X. Song, V.S. Battaglia, J. Electrochem. Soc. 154 (2007) A1129-A1134.

[8] N. Liu, Z. Lu, J. Zhao, M.T. McDowell, H.-W. Lee, W. Zhao, Y. Cui, Nat. Nanotechnol. 9 
(2014) 187-192.

[9] H. Zhao, W. Yuan, G. Liu, Nano Today, 10 (2015) 193-212.

[10] D. Lin, Z. Lu, P.-C. Hsu, H.R. Lee, N. Liu, J. Zhao, H. Wang, C. Liu, Y. Cui, Energy Environ.

Sci. 8 (2015) 2371-2376.

[11] H. Zhao, N. Yuca, Z. Zheng, Y. Fu, V.S. Battaglia, G. Abdelbast, K. Zaghib, G. Liu, ACS Appl. Mater. Interfaces, 7 (2015) 862-866. 


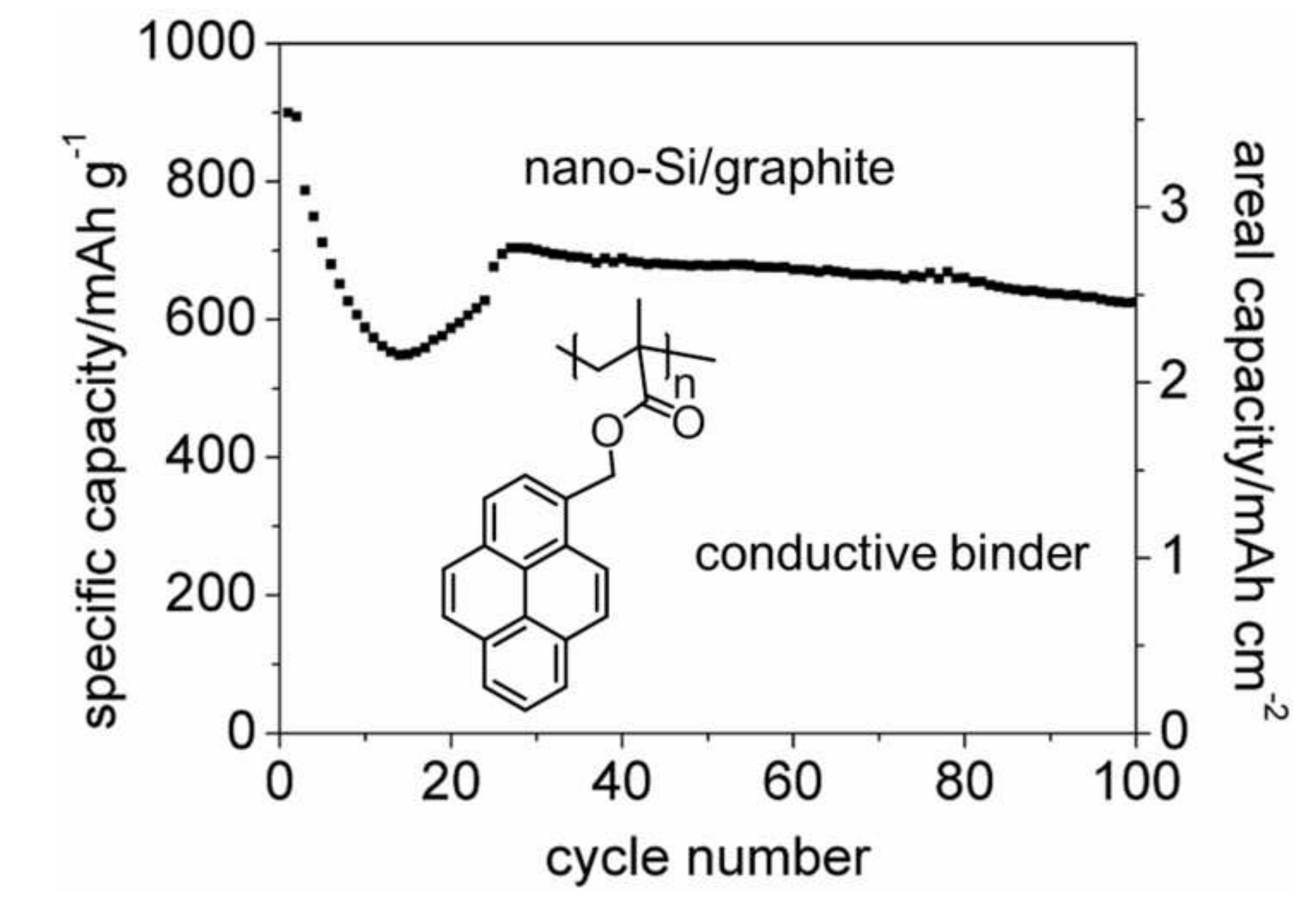

(⿸丆口

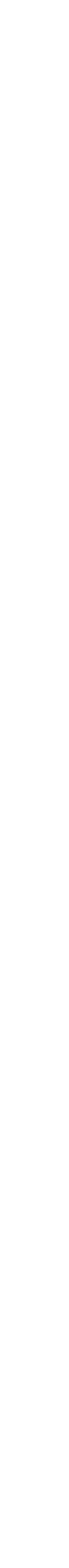

